\title{
Accurate Determination of Junction Temperature in a GaN-Based Blue Light-Emitting Diode using Nonlinear Voltage-Temperature Relation
}

\section{Chibuzo Onwukaeme}

Inha University

Won-Jin Choi

RayIR Corporation

Han-Youl Ryu ( $\sim$ hanryu@inha.ac.kr)

Inha University https://orcid.org/0000-0003-4171-4010

\section{Research Article}

Keywords: Light-emitting diode, Blue LED, GaN, Junction temperature

Posted Date: February 23rd, 2021

DOl: https://doi.org/10.21203/rs.3.rs-223018/v1

License: (1) (1) This work is licensed under a Creative Commons Attribution 4.0 International License.

Read Full License

Version of Record: A version of this preprint was published at Optical and Quantum Electronics on August 14th, 2021. See the published version at https://doi.org/10.1007/s11082-021-03182-6. 


\title{
Accurate determination of junction temperature in a GaN-based
} blue light-emitting diode using nonlinear voltage-temperature

\section{relation}

\author{
Chibuzo Onwukaeme, ${ }^{1}$ Won-Jin Choi, ${ }^{2}$ and Han-Youl Ryu ${ }^{1, *}$ \\ ${ }^{1}$ Department of Physics, Inha University, 100 Inha-ro, Michuhol-gu, Incheon 22212, Korea \\ ${ }^{2}$ RayIR Corporation, LTD, 156 Gwanggyo-ro, Yeongtong-gu, Suwon 16506, Korea \\ *hanryu@inha.ac.kr, http://orcid.org/0000-0003-4171-4010
}

\begin{abstract}
We investigate the junction temperature measurements for GaN-based blue light emitting diodes (LEDs) using nonlinear dependence of the forward voltage $\left(V_{f}\right)$ on temperature. Unlike the conventional linear model of the dependence of $V_{f}$ on temperature, the modeling of the temperature dependent $V_{f}$ with a quadratic function showed good agreements with measured data in the temperature range between 20 and $100{ }^{\circ} \mathrm{C}$. Using the proposed quadratic model, the junction temperature and thermal resistance of the measured LED could be accurately determined as the ambient temperature varied. It was observed that the junction temperature increment remained almost unchanged as the ambient temperature increased from 20 to $80{ }^{\circ} \mathrm{C}$, which could be attributed to the interplay between the decrease in series resistance and the increase in non-radiative recombination with increasing temperature. The presented method for accurate determination of the junction temperature is expected to be advantageously employed for the thermal management of high-power LEDs.
\end{abstract}

Keywords: Light-emitting diode, Blue LED, GaN, Junction temperature 


\section{Introduction}

The demand for light emitting diodes (LEDs) as applied to commercial and residential solid-state lighting, display backlighting, and automobile headlights continues to rise (Chang et al. 2012, Pust et al. 2015, Cho et al. 2017, Bhardwaj et al. 2017). The internal quantum efficiency (IQE) of state-of-the art blue LEDs can exceed 90\% (Hurni et al. 2015, Kuritzky et al. 2018, David et al. 2020). However, the IQE of GaN-based blue LEDs may be reduced significantly at high current density and at high temperature, respectively referred to as current droop and thermal droop (David et al. 2019, Meneghini et al. 2020). Increasing the operation temperature of an LED decreases light output power (LOP) mainly because of the increase in non-radiative recombination with increasing temperature (Schubert 2016). Therefore, thermal management of LEDs has become increasingly important with the development of high power LEDs. Junction temperature $\left(T_{j}\right)$ is an important parameter which could significantly influence IQE, device reliability, and color stability of LEDs. High electrical power consumption by commercial high-power LEDs results in device heating, making $T_{j}$ an essential figure of merit that requires a reliable method of measurement. Because of the importance of thermal management in LEDs, $T_{j}$ of GaN-based LEDs has been intensively studied.

In addition to LOP, the forward voltage $\left(V_{f}\right)$ of GaN-based LEDs has been demonstrated to show strong dependence on temperature (Xi et al. 2004, Ryu et al. 2005). $V_{f}$ decreases with increasing temperature mainly because of the reduction in the energy band gap and series resistance (Varshini 1967, Xi et al. 2005). The large variation of $V_{f}$ with temperature can provide a convenient and reliable method to determine $T_{j}$ (Xi et al. 2004, Ryu et al. 2005, Keppens et al. 2008, Feng et al. 2012). This forward voltage method has been known to be more accurate than the emission-peak-shift method (Xi et al. 2005). In the forward voltage 
method, the temperature coefficient of voltage $(K)$, which is defined as the derivative of forward voltage with respect to temperature, is measured firstly. Then, $T_{j}$ is determined by dividing the voltage difference between pulsed and continuous-wave $(\mathrm{CW})$ operation by the measured $K$ value. Up to now, $K$ was mostly regarded to be constant independent of temperature in determining $T_{j}$ of LEDs, assuming almost linear dependence of $V_{f}$ on temperature. However, $K$ can be a strong function of temperature because of nonlinear dependence of $V_{f}$ on temperature, which should be considered in determining $T_{j}$.

In this paper, we report on the method for accurate determination of $T_{j}$ of a GaN-based blue LED package in the temperature range between 20 to $100{ }^{\circ} \mathrm{C}$ using nonlinear temperature dependence of the forward voltage. It will be shown that $V_{f}$ can be fit quite well with a quadratic function of the temperature, and hence the $K$ value depends linearly on the temperature. Using the obtained $K$-factor, the junction temperature increment $\left(\Delta T_{j}\right)$ relative to the ambient temperature was determined. In addition, thermal resistance $\left(R_{t h}\right)$ of the measured LED package was determined using $\Delta T_{j}$ and dissipated electrical power. We compare the temperature dependence of $T_{j}$ and $R_{t h}$ for the constant and variable $K$ values, and show the importance of using nonlinear temperature dependence of $V_{f}$ for the accurate characterization of thermal properties of LEDs.

\section{Theory}

The relation between $V_{f}$ and injection current $(I)$ at temperature $T$ of a diode is given by the Shockley equation that includes a series resistance term (Xi et al. 2005):

$$
I=I_{s}\left[\exp \left(\frac{e V_{f}-e I R_{s}}{n k T}\right)-1\right],
$$

where $I_{S}$ is the saturation current, $R_{S}$ is the series resistance, and $n$ is the diode ideality factor. 
Solving Eq. (1) for the case of $e V_{f} \square k T, V_{f}$ is expressed as

$$
V_{f}=V_{j}+I R_{s}=\frac{n k T}{e} \ln \left(\frac{I}{I_{s}}\right)+I R_{s},
$$

where $V_{j}$ is the junction voltage. By taking the derivative of $V_{f}$ with respect to $T$ in Eq. (2), the temperature coefficient of voltage, $K$ is obtained:

$$
K \equiv \frac{d V_{f}}{d T}=\frac{d V_{j}}{d T}+I \frac{d R_{s}}{d T},
$$

According to Refs. (Xi et al. 2005, Feng et al. 2012, Chen et al. 2017 el al.), Eq. (3) can be written as

$$
K=\frac{d V_{j}}{d T}-\frac{1}{2} \frac{E_{a}+2 S k T}{k T^{2}} I R_{s}
$$

where $E_{a}$ is the acceptor activation energy of p-GaN and $S$ is the exponent of temperature dependence in the carrier mobility model.

In Eq. (4), $d V_{j} / d T$ was found to be weakly dependent on the temperature, which showed an almost constant value around $-1.5 \mathrm{mV} / \mathrm{K}$ for InGaN blue LEDs (Xi et al. 2005, Meyaard et al. 2013). The second summand of the right hand side of Eq. (4), which corresponds to the contribution of the series resistance, can be strongly dependent on the temperature and current. This term has complicated temperature dependence as $R_{s}$ is also dependent on the temperature. Up to now, $K$ of LEDs was mostly regarded to be constant independent of temperature and current (Xi et al. 2004, Keppens et al. 2008, Meyaard et al. 2013, Kim et al. 2016, Chen et al. 2017,). However, this assumption is valid only for the cases of low injection current and a limited temperature range, suggesting that the temperature dependence of $K$ should be considered in determining $T_{j}$ for LEDs operating at high current.

Using the definition of $K$ in Eq. (3), the difference in $V_{f}$ between $\mathrm{CW}$ and pulsed operation is expressed as 


$$
\Delta V_{f}=\int_{T_{a}}^{T_{j}} K d T
$$

where $T_{a}$ is the ambient temperature. Eq. (5) is based on the assumption that the temperature of LEDs corresponds to $T_{j}$ and $T_{a}$ for the $\mathrm{CW}$ and pulsed operation, respectively. It should be noted that both $\Delta V_{f}$ and $K$ are negative quantities. If the voltage-temperature $(V-T)$ relation was assumed to be linear, $K$ would be constant. In that case, $\Delta T_{j}$ is simply given by

$$
\Delta T_{j} \equiv T_{j}-T_{a}=\frac{\Delta V_{f}}{K}
$$

If $V_{f}$ is quadratically proportional to the temperature as will be shown experimentally, $K$ can be written as

$$
K=a T+b,
$$

where $a$ and $b$ are fitting parameters that can be obtained by the fit of measured $V$-T relation. Using Eqs. (5) and (7), the following quadratic equation for $T_{j}$ is obtained:

$$
a T_{j}^{2}+2 b T_{j}-\left(a T_{a}^{2}+2 b T_{a}+2 \Delta V_{f}\right)=0
$$

Solving Eq. (8) yields $T_{j}$ for given $T_{a}$ and $\Delta V$. In addition, thermal resistance of the LED package can be calculated using the measured $T_{j}$ and light output power, $P$ :

$$
R_{t h}=\frac{\Delta T_{j}}{I V_{f}-P}
$$

\section{Experiments}

The epitaxial layers of an LED used for this study were grown on a $c$-plane sapphire substrate by metal-organic chemical vapor deposition. The layer structure consisted of a Sidoped n-GaN layer, InGaN/GaN multiple-quantum-well active region, a Mg-doped p-AlGaN electron-blocking layer, and a Mg-doped p-GaN contact layer. The peak emission wavelength was $\sim 450 \mathrm{~nm}$ at $20{ }^{\circ} \mathrm{C}$. The LED chip was fabricated as a lateral-injection structure with the 
chip dimension of $650 \mu \mathrm{m} \times 650 \mu \mathrm{m}$. The fabricated LED chip was encapsulated with epoxy resin and mounted in a ceramic package as a type of surface-mount device. Then, the LED package was soldered on a metal PCB and the temperature was controlled by a thermoelectric cooler.

The optical and electrical characteristics of an LED sample were measured as the injection current increased up to $150 \mathrm{~mA}$ for a fixed ambient temperature $\left(T_{a}\right)$ from 20 to $100{ }^{\circ} \mathrm{C}$, and the LOP versus current $(L-I)$ and $V_{f}$ versus current $(V-I)$ relations were obtained for each temperature. The LED sample was operated under CW and pulsed current injection for a given $T_{a}$. For the pulsed operation, the pulse width and the duty cycle were $0.1 \mathrm{~ms}$ and the duty cycle of $1 \%$, respectively, which is expected to have negligible effect on temperature rise of the LED junction.

Figure 1 shows $L-I$ curves of the measured LED sample under CW operation for $T_{a}$ of 20, $40,60,80$, and $100{ }^{\circ} \mathrm{C}$. The LOP increased sublinearly with injection current, and it decreased slowly with increasing temperature. Figure 2 shows $V$-I curves of the measured LED sample. In Fig. 2(a), $V-I$ curves under pulsed operation are shown for various $T_{a}$ between 20 and 100 ${ }^{\circ} \mathrm{C}$. It is observed that $V_{f}$ decreased with increasing temperatures. In Fig. 2(b), V-I curves under pulsed and $\mathrm{CW}$ operation are compared when the ambient temperature is $20{ }^{\circ} \mathrm{C} . V_{f}$ under pulsed operation was higher than that under $\mathrm{CW}$ operation as a result of the difference in the LED temperature between pulsed and $\mathrm{CW}$ operation. The voltage difference, $\Delta V_{f}$ between pulsed and CW operation is used to determine $T_{j}$ using Eq. (6) or (8).

\section{Results and Discussion}

An accurate thermal measurement evaluation requires an ideal fit to the forward voltage versus temperature $(V-T)$ relation. Figure 3(a) shows $V_{f}$ under pulsed operation as a function 
of $T_{a}$ when the injection current was $100 \mathrm{~mA}$. Because of the pulsed operation, it can be regarded that $T_{a}$ is the same as $T_{j}$ in Fig. 3(a). The linear fit (red line) and a quadratic fit (blue line) to the measured data are also shown in Fig. 3(a). As mentioned before, the dependence of $V_{f}$ on temperature has often been modelled with a linear function (Xi et al. 2004). However, inspection of Fig. 3(a) shows that the linear fit did not agree well with the measured data. In fact, a linear fit implies a constant slope and consequently a single value of the series resistance in the entire temperature, which in reality is not the case. Increasing the temperature activates the acceptor atoms in the $p$ doped region resulting in a changing series resistance. For this reason, one would expect a varying slope. By modelling the temperature dependent forward voltage with a quadratic function, $V_{f}=\alpha T^{2}+\beta T+\gamma$, a nearly ideal fit which agreed well with the experimental data was demonstrated. The $R^{2}$ value for the linear and quadratic fit was 0.9936 and 0.9998 , respectively.

The temperature coefficient of voltage, $K$ is the slope of the $V-T$ relation, which is obtained by the derivative of the fit function. For the linear fitting, $K$ was constant value of $3.91 \mathrm{mV} / \mathrm{K}$ at $100 \mathrm{~mA}$. For the quadratic fitting, $K$ is given by a temperature dependent function, $K=2 \alpha T_{a}+\beta$. Figure 3(b) shows the variation of $K$ with $T_{a}$ for the quadratic fit. In this case of 100-mA injection current, $\alpha$ and $\beta$ were obtained to be $-8.74 \times 10^{-6} \mathrm{~V} / \mathrm{K}^{2}$ and $4.96 \times 10^{-3} \mathrm{~V} / \mathrm{K}$, respectively. It has been shown that the slope of the $V$ - $T$ curve decreases as the series resistance is reduced (Meyaard et al. 2013). Therefore, the negative value of the slope $\alpha$ with increasing temperature implies a decreasing value of the series resistance with temperature.

The junction temperature increment, $\Delta T_{j}$ can be determined using Eq. (6) and Eq. (8) for the linear and quadratic $V$ - $T$ fit, respectively. Figure 4(a) shows the variation of $\Delta T_{j}$ as a function of $T_{a}$ for $100-\mathrm{mA}$ injection current. The result reveals slowly varying $\Delta T_{j}$ for the 
quadratic $V$ - $T$ fitting and rapidly decreasing $\Delta T_{j}$ for the linear fitting. The conventional linear $V$ - $T$ fit could lead to large errors in determining $\Delta T_{j}$ of LEDs at low and high $T_{a} . \Delta T_{j}$ for the quadratic $V$ - $T$ fitting varied between 19 to $23 \mathrm{~K}$ as $T_{a}$ increased from 20 to $100{ }^{\circ} \mathrm{C}$. Increase in temperature will increase the activation of acceptor atoms in the p-region thereby reducing the series resistance contribution to the junction heating. On the contrary, nonradiative recombination via SRH recombination and Auger recombination increases at elevated temperatures, resulting in the increase of heat generation at the junction. The slowly varying $\Delta T_{j}$ observed in Fig. 4(a) can be attributed to the interplay between the contributions of the series resistance of the p-region and the nonradiative recombination to the junction heating.

With the obtained results of $\Delta T_{j}$, LOP, and $V_{f}$ under CW operation, $R_{t h}$ was calculated using Eq. (9). Figure 4(b) shows the variation of $R_{t h}$ with $T_{a}$ for the linear and quadratic $V-T$ fitting at $100-\mathrm{mA}$ injection current. The dependence of $R_{t h}$ on $T_{a}$ is similar to that of $\Delta T_{j}$ on $T_{a}$ in Fig. 4(a) for both the linear and quadratic fitting. As the temperature increased from 20 to $100{ }^{\circ} \mathrm{C}, R_{t h}$ decreased from 159 to $98 \mathrm{~K} / \mathrm{W}$ for the linear fitting whereas it slowly varied from 126 to $147 \mathrm{~K} / \mathrm{W}$ for the quadratic fitting. As in the case of $\Delta T_{j}$, the linear fitting of $V-T$ relation could lead to incorrect information on $R_{t h}$. Since $R_{t h}$ is strongly influenced by thermal conductivity of thermal interface materials (TIMs), the slowly varying behavior of $R_{t h}$ with temperature can be attributed to the temperature dependence of TIMs in the LED package.

In addition, we evaluated $\Delta T_{j}$ for other injection currents using the quadratic fitting of $V$ $T$ relations and Eq. (8). Figure 5 shows $\Delta T_{j}$ as a function injection current for $T_{a}$ of $20,40,60$, and $80{ }^{\circ} \mathrm{C} . \Delta T_{j}$ increased nearly linearly with increasing current for all temperatures. This linear relationship has also been reported in previous works on the junction temperature of LEDs (Xi et al. 2005, Feng et al. 2012, Kim et al. 2020). The slope of $\Delta T_{j}$ versus current was calculated to be $\sim 0.24 \mathrm{~K} / \mathrm{A}$. In addition, only slight difference in $\Delta T_{j}$ was observed at a given 
injection current for all temperatures from 20 to $80{ }^{\circ} \mathrm{C}$. As the temperature increases, the series resistance decreases whereas nonradiative recombination increases. These counteractive effects are believed to result in the insensitivity of $\Delta T_{j}$ to ambient temperatures.

\section{Conclusion}

We have reported on the determination of the junction temperature of GaN blue LED using a non-linear modelling of the forward voltage dependence on temperature. It was found that the temperature dependence of $V_{f}$ was fitted ideally with a quadratic function in contrast to the linear dependence in previous studies. This resulted in decreasing values of the temperature coefficient of voltage, which is an implication of the variation of the series resistance with temperature. Using the quadratic model, $\Delta T_{j}$ of the LED could be accurately determined as the ambient temperature increased from 20 to $100{ }^{\circ} \mathrm{C}$. On the contrary, when the conventional linear fit of the voltage-temperature relation was employed, large errors could occur in determining $\Delta T_{j}$. As the temperature varied between 20 to $80{ }^{\circ} \mathrm{C}, \Delta T_{j}$ was found to change only slightly with the ambient temperature, which could be explained by the opposite temperaturedependent contribution of series resistance and nonradiative recombination to heat generation. The presented analysis model on the voltage-temperature relation is expected to provide the method to accurately determine the junction temperature of LEDs, and thereby contribute to the thermal management of high-power LEDs.

\section{Acknowledgments}

This work was supported by the National Research Foundation of Korea (NRF) grant funded by the Ministry of Science and ICT (NRF-2019R1A2C1010160) and the Ministry of Education (NRF-2016R1D1A1B03932092). 


\section{Declarations}

\section{Funding}

National Research Foundation of Korea Grant

(NRF-2019R1A2C1010160, NRF-2016R1D1A1B03932092)

\section{Conflicts of interest/Competing interests}

Not applicable

\section{Data availability}

The datasets generated during and/or analysed during the current study are available from the corresponding author on reasonable request. 


\section{References}

Bhardwaj, J., Cesaratto, J.M., Wildeson, I.H., Choy, H., Tandon, A., Soer, W.A., Schmidt, P.J., Spinger, B., Deb,P., Shchekin, O.B., Götz, W.: Progress in high-luminance LED technology for solid-state lighting. Phys. Status Solidi A 214, 1600826 (2017). https://www.doi.org/10.1002/pssa.201600826

Chang, M.H., Das, D., Varde, P.V., Pecht, M.: Light emitting diodes reliability review. Microelectronics Reliability 52, 762-782 (2012). https://www.doi.org/10.1016/j.microrel.2011.07.063

Cho, J., Park, J.H., Kim, J.K., Schubert, E.F.: White light-emitting diodes: History, progress, and future. Laser Photonics Rev. 11, 1600147 (2017). https://www.doi.org/10.1002/lpor.201600147

Chen, B.C., Chen, K.H., Yu, J.W., Ho, C.W., Wen, M.Y.: Analysis of junction temperatures for groups III-V semiconductor materials of light-emitting diodes. Opt. Quant. Electron. 49, 183 (2017). https://www.doi.org/ 10.1007/s11082-017-1015-6

David, A., Young, N.G., Lund, C., Craven, M.D.: Review-The physics of recombinations in III-nitride emitters. ECS J. Solid State Sci. Technol. 9, 016021 (2020). https://www.doi.org/ 10.1149/2.0372001JSS

David, A., Young, N.G., Lund, C., Craven, M.D.: Thermal droop in high-quality InGaN LEDs. Appl. Phys. Lett. 115, 223502 (2019). https://doi.org/10.1063/1.5124123

Feng, M.X., Zhang, S.M., Jiang, D.S., Liu, J.P., Wang, H., Zeng, C., Li, Z.C., Wang, H.B., Wang, F., Yang, H.: Thermal characterization of GaN-based laser diodes by forwardvoltage method. J. Appl. Phys. 111, 094513 (2012). https://www.doi.org/10.1063/1.4716003 
Hurni, A., David, A., Cich, M.J., Aldaz, R.I., Ellis, B., Huang, K., Tyagi, A., DeLille, R.A., Craven, M.D., Steranka, F.M.: Bulk GaN flip-chip violet light-emitting diodes with optimized efficiency for high-power operation. Appl. Phys. Lett. 106, 031101 (2015). https://doi.org/10.1063/1.4905873

Keppens, A., Ryckaert, W.R., Deconinck, G., Hanselaer, P.: High power light-emitting diode junction temperature determination from current-voltage characteristics. J. Appl. Phys. 104, 093104 (2008). https://doi.org/10.1063/1.3009966

Kim, D.S., Han, B., Effect of junction temperature on heat dissipation of high power light emitting diodes. J. Appl. Phys. 119, 125104 (2016). https://doi.org/10.1063/1.4944800

Kim, D.Y., Park, J., Cho, J., Kim, J.K.: Counter-intuitive junction temperature behavior in AlGaN-based deep-ultraviolet light-emitting diodes. AIP Advances 10, 045135 (2020). https://doi.org/10.1063/5.0005473

Kuritzky, L.Y., Weisbuch, C., Speck, J.S.: Prospects for 100\% wall-plug efficiency III-nitride LEDs. Opt. Express 26, 16600-16608 (2018). https://doi.org/10.1364/OE.26.016600

Meneghini, M., Santi, C.D., Tibaldi, A., Vallone, M., Bertazzi, F., Meneghesso, G., Zanoni, E., Goano, M.: Thermal droop in III-nitride based light-emitting diodes: Physical origin and perspectives. J. Appl. Phys. 127, 21102 (2020). https://doi.org/10.1063/5.0005874

Meyaard, D.S., Cho, J., Schubert, E.F., Han, S.H., Kim, M.H., Sone, C.: Analysis of the temperature dependence of the forward voltage characteristics of GaInN light-emitting diodes. Appl. Phys. Lett. 103, 121103 (2013). http://dx.doi.org/10.1063/1.4821538

Ryu, H.Y., Ha, K.H., Chae, J.H., Nam, O.H., Park, Y.J.: Measurement of junction temperature in GaN-based laser diodes using voltage-temperature characteristics. Appl. Phys. Lett. 87, 093506 (2005). https://doi.org/10.1063/1.2037201

Pust, P., Schmidt, P.J., Schnick, W.: A revolution in lighting. Nat. Mater. 14, 454-458 (2015). 
Schubert, E.F.: Light-emitting diodes. 2nd ed. Cambridge University Press, Cambridge, (2006)

Xi, Y., Gessmann, T., Xi, J., Kim, J.K., Shah, J.M., Schubert, E.F., Fischer, A.J., Crawford, M.H., Bogart, K.H.A., Allerman, A.A.: Junction temperature in ultraviolet lightemitting diodes,” Jpn. J. Appl. Phys. 44, 7260-7266 (2005). https://doi.org/10.1143/JJAP.44.7260

Xi. Y., Schubert, E.F.: Junction-temperature measurement in GaN ultraviolet light-emitting diodes using diode forward voltage method. Appl. Phys. Lett. 85, 2163-2165, (2004). https://doi.org/10.1063/1.1795351

Varshni, Y.P.: Temperature dependence of the energy gap in semiconductors. Physica 34, 149 (1967). 


\section{Figures}

Fig. 1. Light output power (LOP) as a function of injection current under $\mathrm{CW}$ operation for the ambient temperature $\left(T_{a}\right)$ of $20,40,60,80$, and $100{ }^{\circ} \mathrm{C}$.

Fig. 2. (a) Forward voltage $\left(V_{f}\right)$ as a function of injection current $(I)$ under pulsed operation for $T_{a}$ of $20,40,60,80$, and $100{ }^{\circ} \mathrm{C}$. (b) The $V_{f}$ versus $I$ relations under pulsed and CW operation are compared when $T_{a}$ is $20{ }^{\circ} \mathrm{C}$.

Fig. 3. (a) $V_{f}$ under pulsed operation as a function of $T_{a}$ for the injection current of $100 \mathrm{~mA}$. The red and blue lines respectively correspond to the linear and quadratic fit to the measured data. (b) Temperature coefficient of voltage $(K)$ as a function of $T_{a}$ for the quadratic fit at $100 \mathrm{~mA}$.

Fig. 4. (a) Junction temperature increment $\left(\Delta T_{j}\right)$ and (b) thermal resistance $\left(R_{t h}\right)$ as a function of $T_{a}$ at $100-\mathrm{mA}$ injection current for the linear and quadratic fit of the voltagetemperature relation.

Fig. 5. $\Delta T_{j}$ as a function injection current for $T_{a}$ of $20,40,60$, and $80{ }^{\circ} \mathrm{C}$. 
Fig. 1

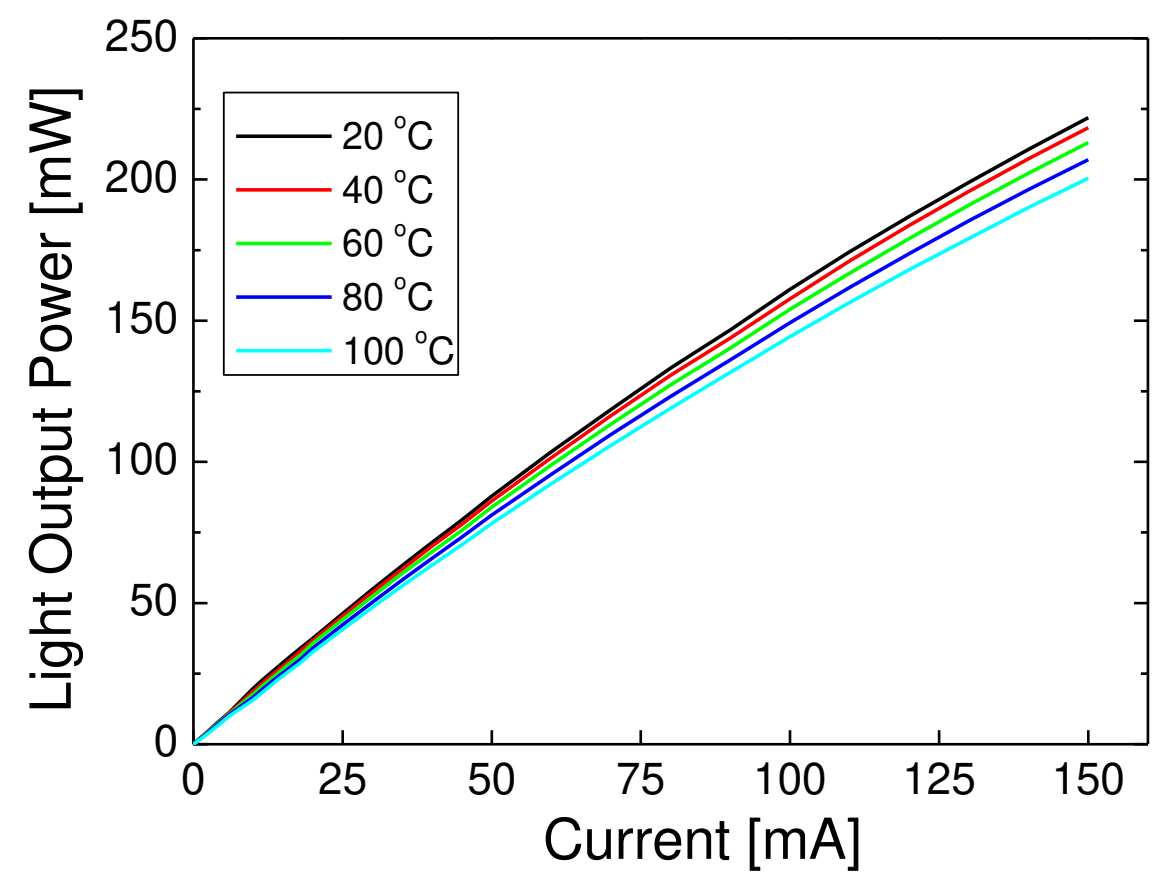


FIG. 2
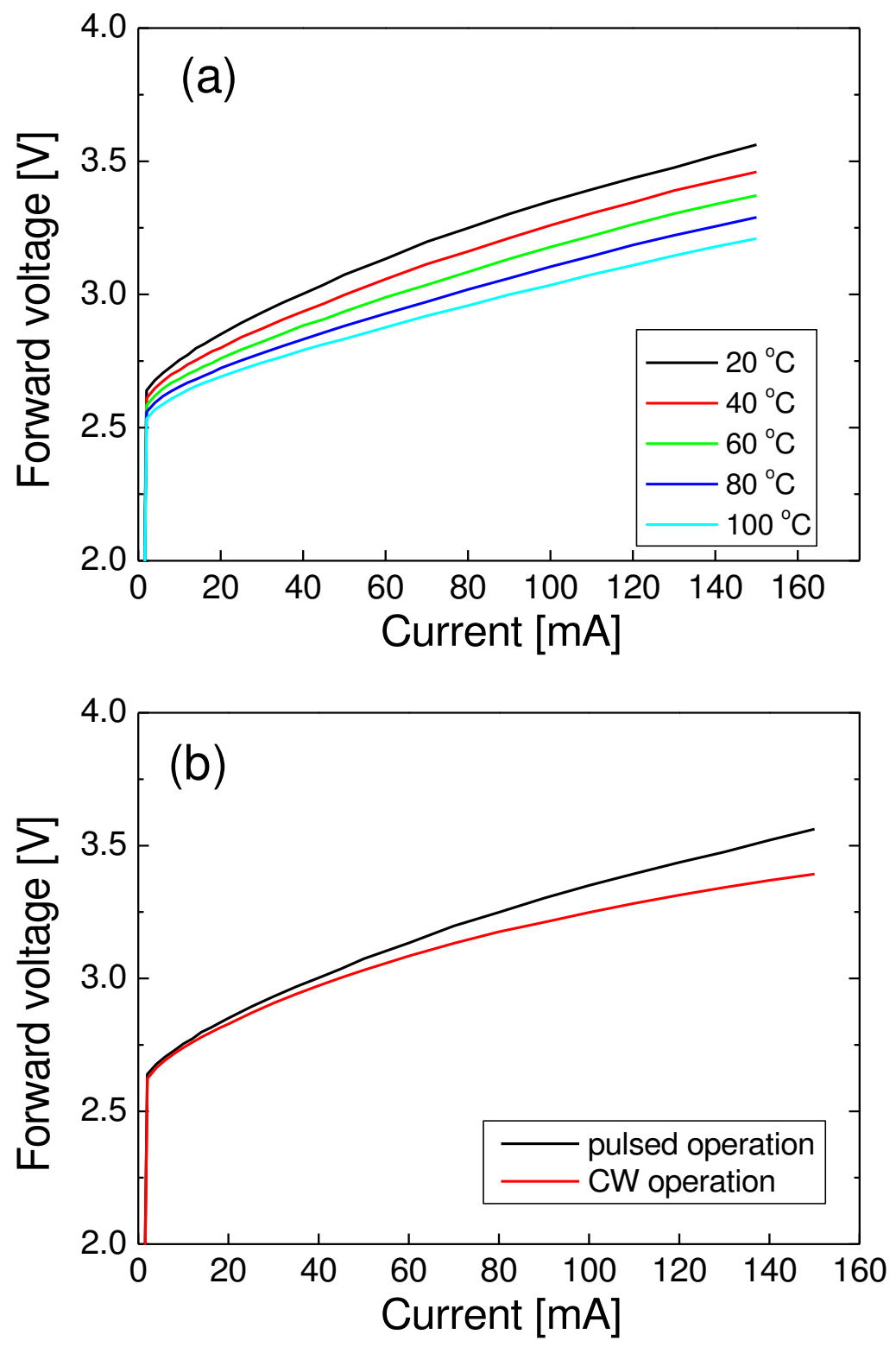
FIG. 3
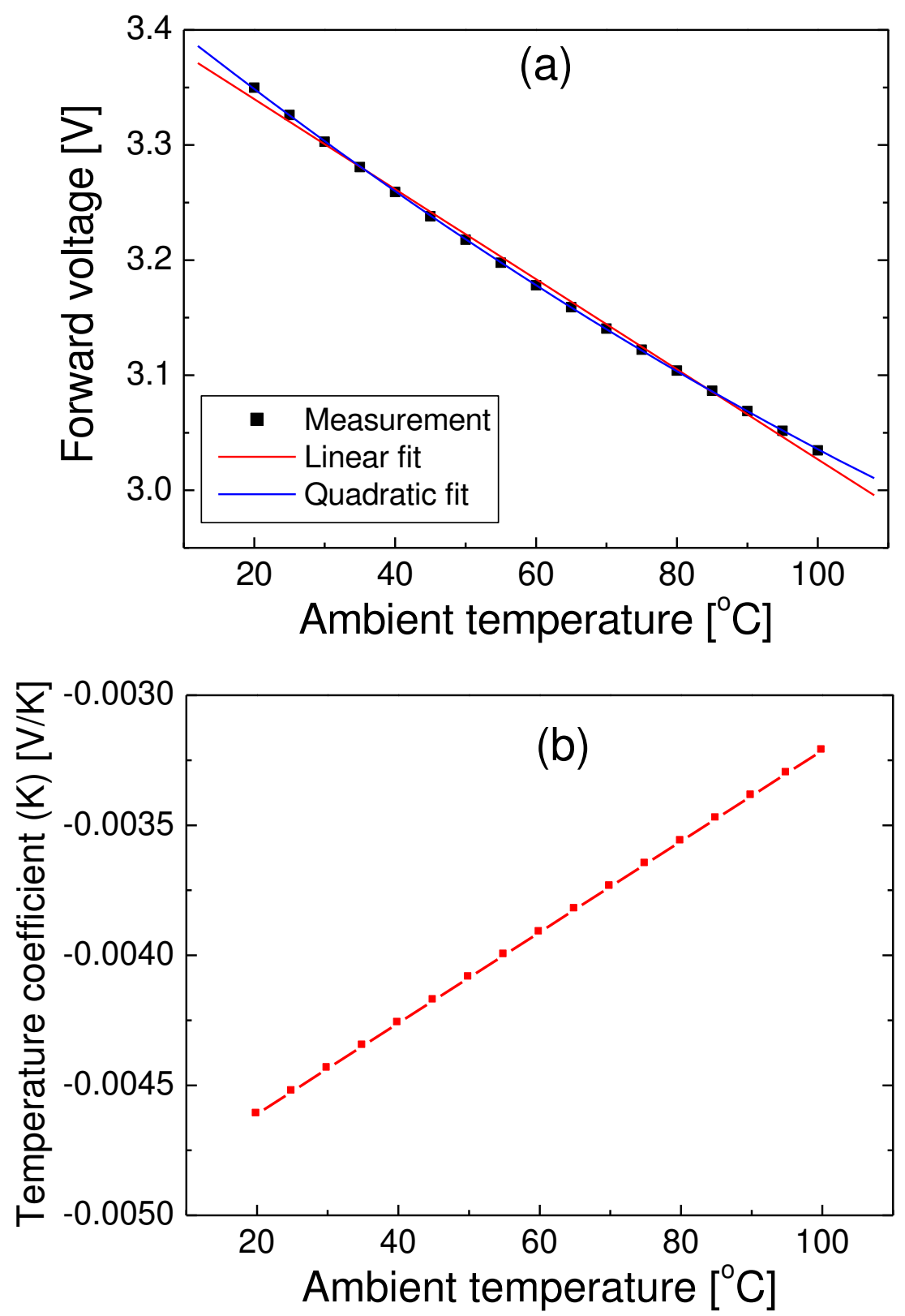
FIG. 4
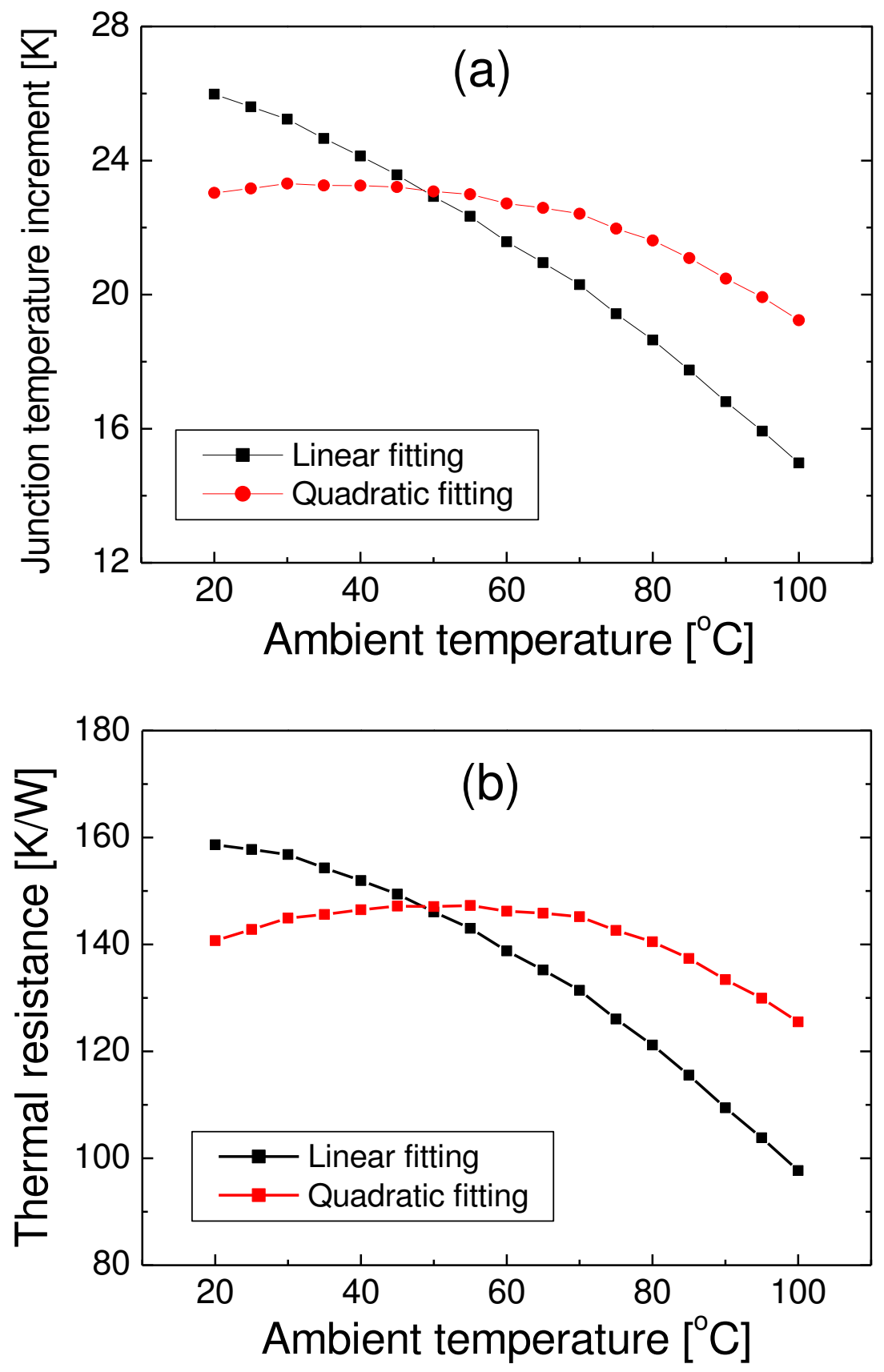
FIG. 5

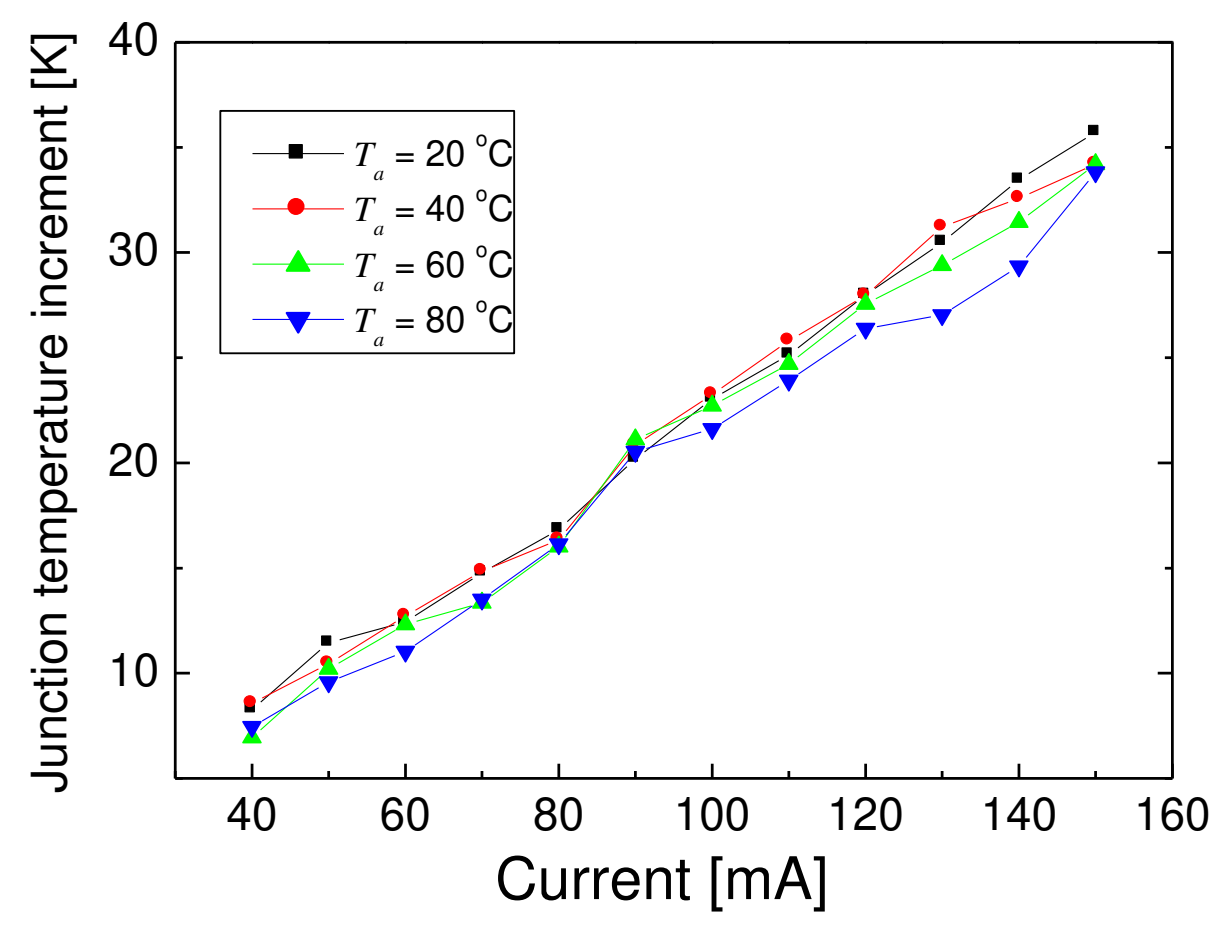


Figures

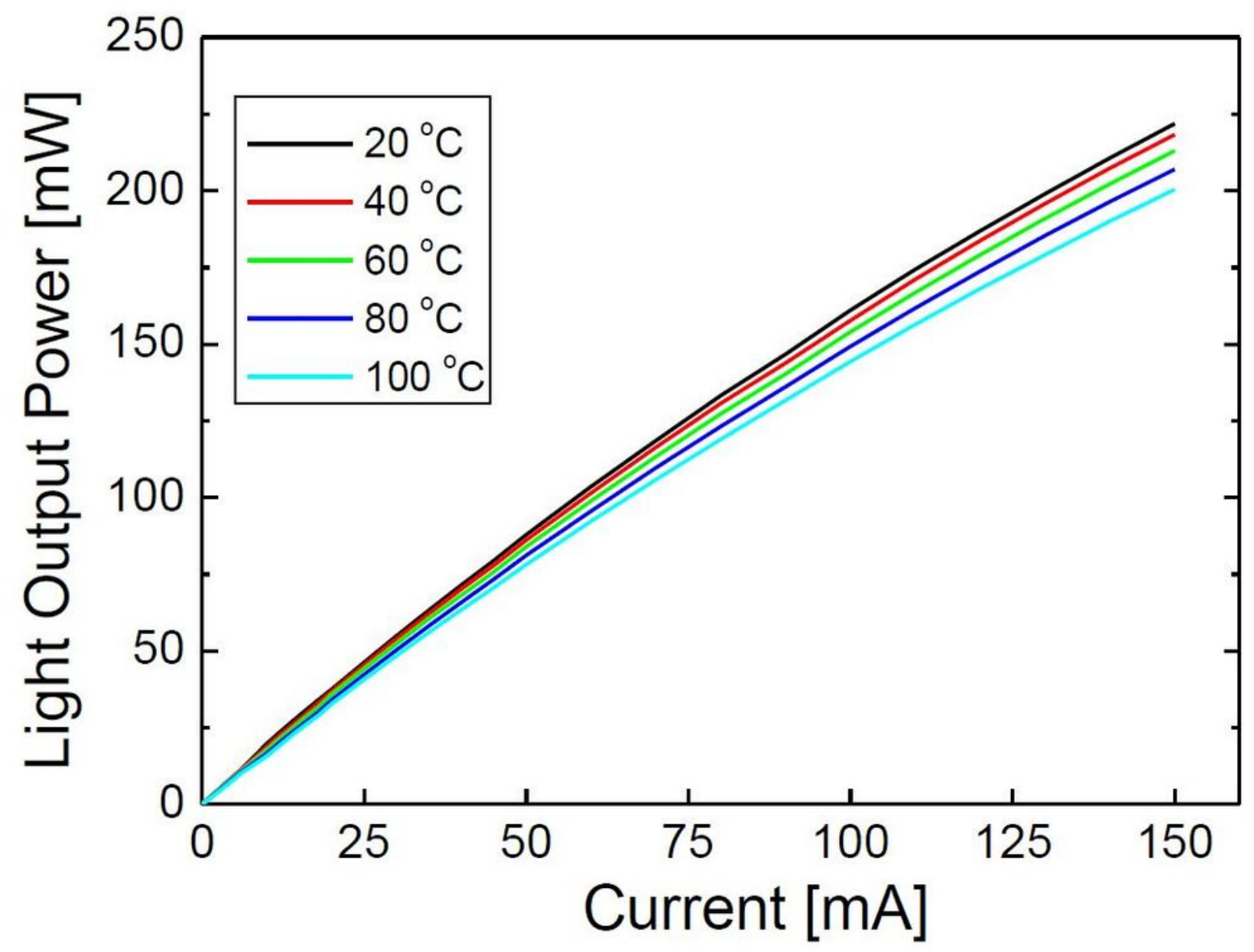

Figure 1

Light output power (LOP) as a function of injection current under $\mathrm{CW}$ operation for the ambient temperature (Ta) of $20,40,60,80$, and $100 \mathrm{oC}$. 

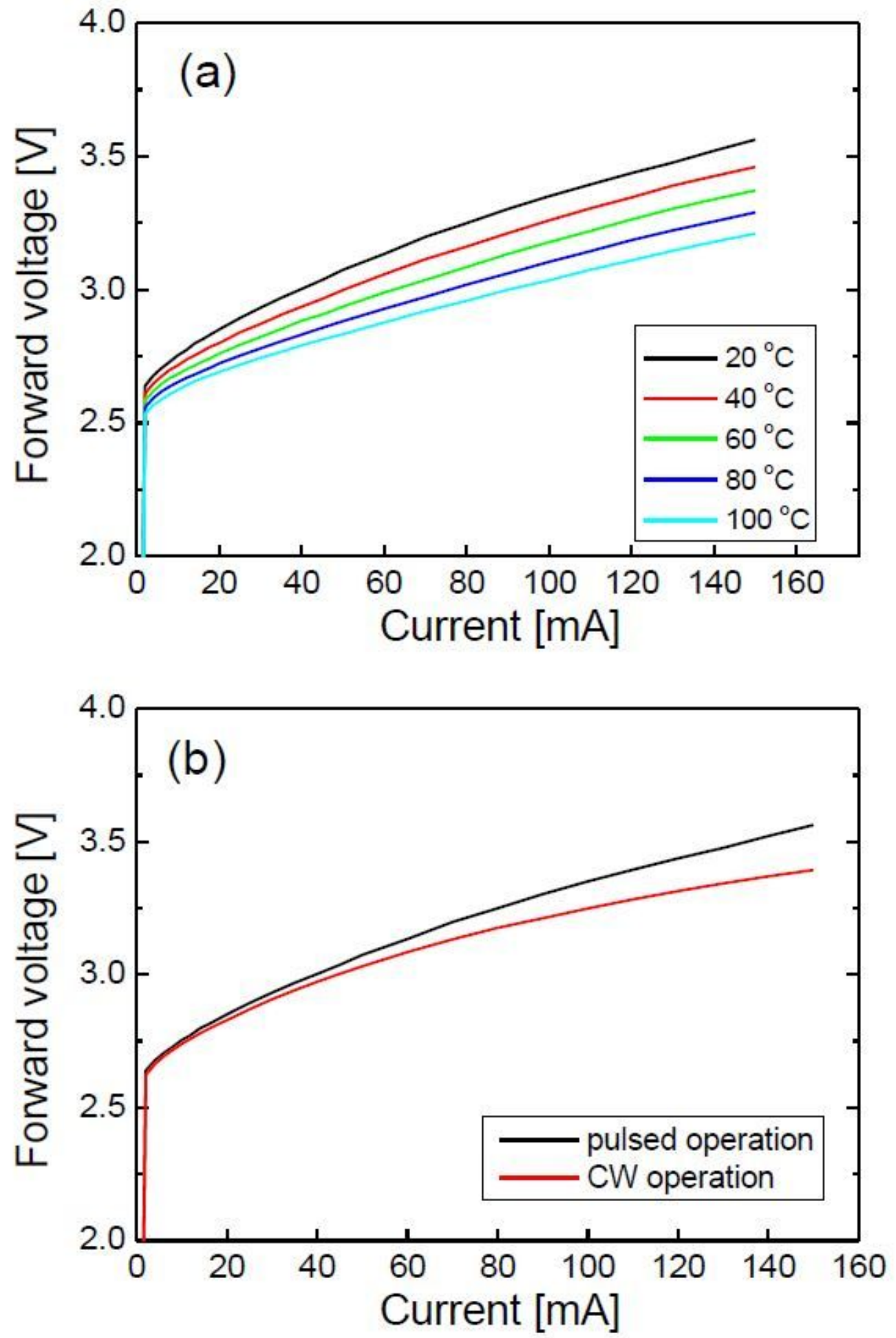

Figure 2

(a) Forward voltage (Vf) as a function of injection current (I) under pulsed operation for Taof 20, 40,60, 80 , and $100 \mathrm{oC}$. (b) The Vfversus I relations under pulsed and $\mathrm{CW}$ operation are compared when Tais $200 \mathrm{C}$. 

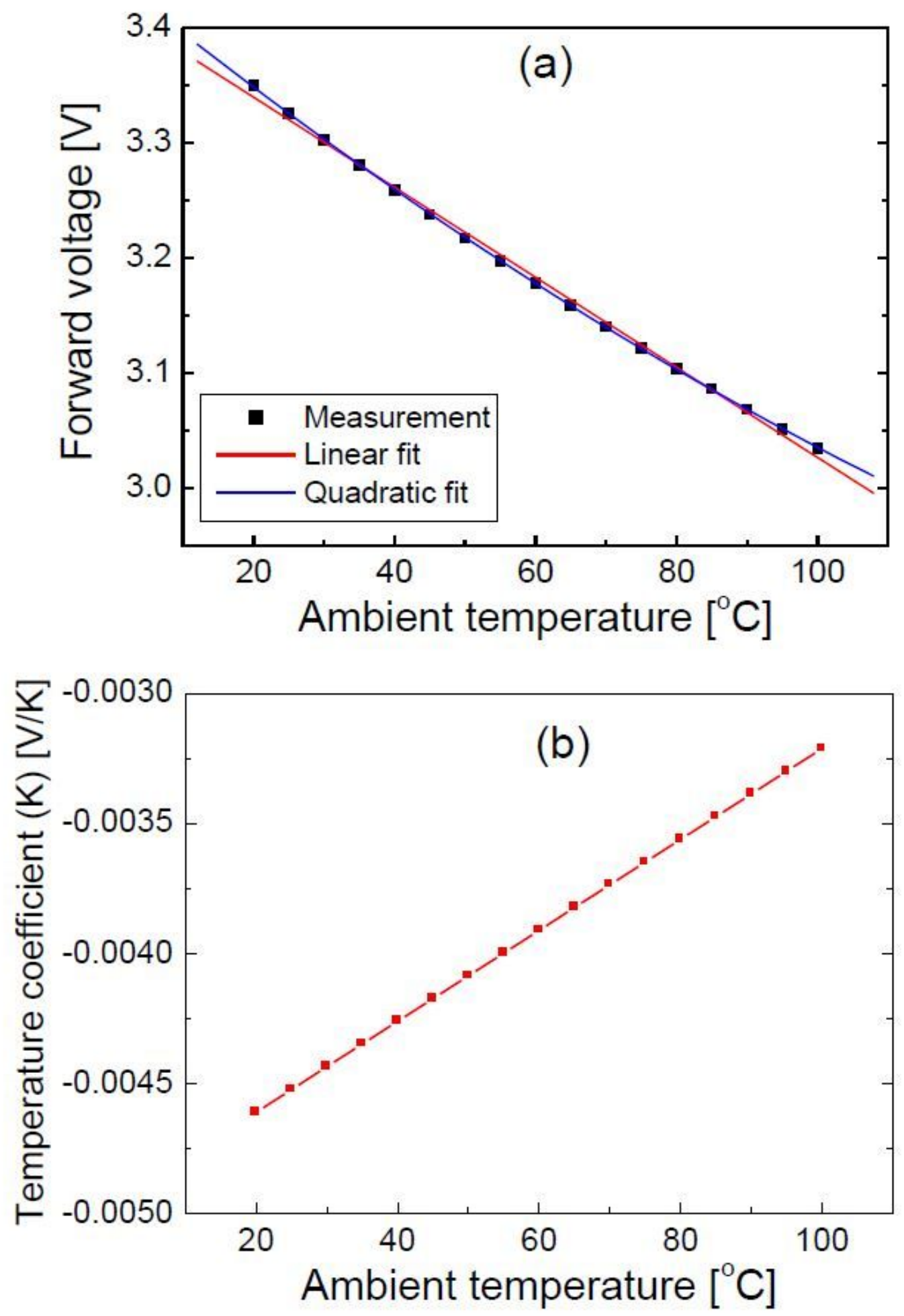

Figure 3

(a) Vf under pulsed operation as a function of Ta for the injection current of $100 \mathrm{~mA}$. The red and blue lines respectively correspond to the linear and quadratic fit to the measured data. (b) Temperature coefficient of voltage $(\mathrm{K})$ as a function of Ta for the quadratic fit at $100 \mathrm{~mA}$. 

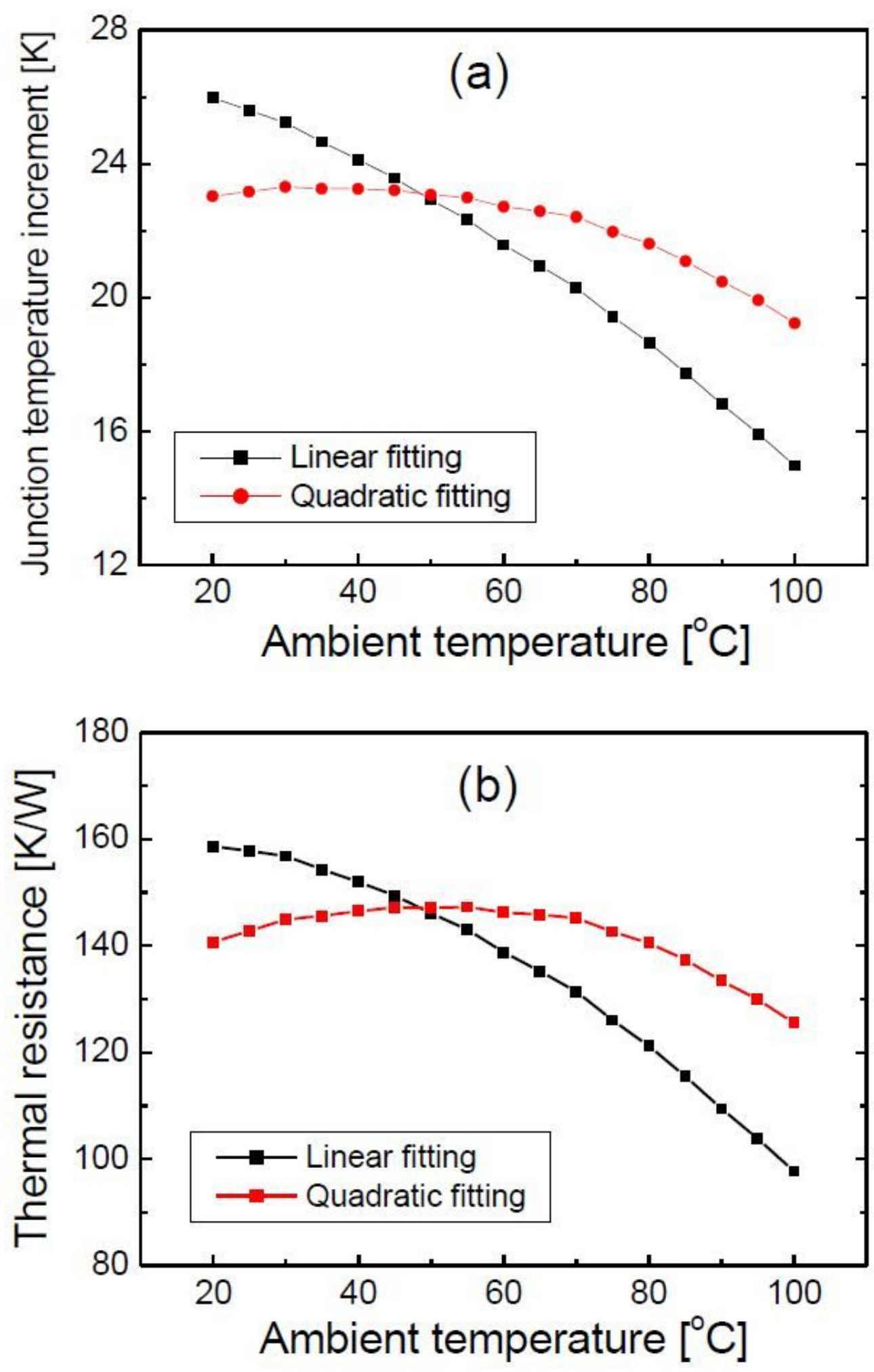

Figure 4

(a) Junction temperature increment $(\Delta \mathrm{Tj})$ and (b) thermal resistance (Rth) as a function of Ta at 100-mA injection current for the linear and quadratic fit of the voltage- temperature relation. 


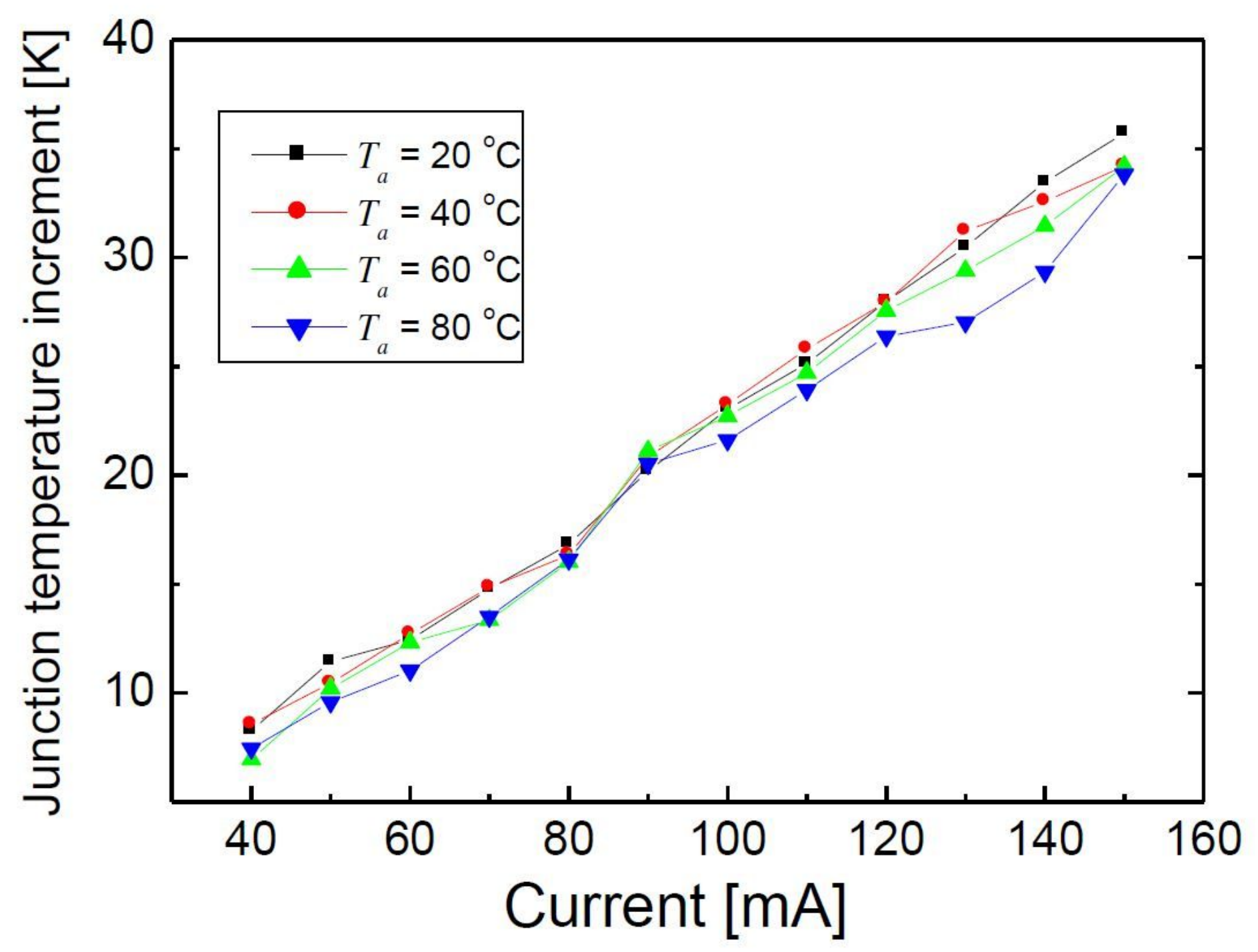

Figure 5

$\Delta \mathrm{Tj}$ as a function injection current for Ta of $20,40,60$, and $80 \mathrm{oC}$. 